\title{
Functional Histology of the Macula Flava in the Human Vocal Fold - Part 1: Its Role in the Adult Vocal Fold
}

\author{
Kiminori Sato Hirohito Umeno Tadashi Nakashima \\ Department of Otolaryngology-Head and Neck Surgery, Kurume University School of Medicine, Kurume, Japan
}

\section{Key Words}

Macula flava $\cdot$ Human vocal fold $\cdot$ Larynx $\cdot$ Extracellular matrix $\cdot$ Vocal fold stellate cell $\cdot$ Aging

\begin{abstract}
Objective: This study aims to clarify the role of the maculae flavae (MFe) in the human adult vocal fold mucosa (VFM). Methods: Our current results concerning MFe in the human adult VFM are summarized. Results: MFe were found to be composed of dense masses of vocal fold stellate cells (VFSCs) and extracellular matrices (EM), such as fibrous proteins and glycosaminoglycans, which are essential for the EM in the human VFM. VFSCs in the MFe demonstrated marked morphologic differences from conventional fibroblasts. They were irregular and stellate in shape and possessed slender cytoplasmic processes. They had well-developed intracellular organelles. A number of vesicles were present at the periphery of the cytoplasm. They constantly synthesized EM. The VFSCs possessed lipid droplets and stored vitamin A. VFSCs formed an independent cell category of cells in the human VFM. The VFSCs in aged adult MFe decreased their activity, and had abnormal metabolism. Conclusion: Human MFe including VFSCs seem to be involved in the metabolism of EM which are essential for the viscoelasticity of the lamina propria of the VFM, and to be responsible for maintaining
\end{abstract}

the characteristic layered structure of the human VFM. Agerelated changes in VFSCs were found to influence the metabolism of EM in the VFM. Copyright $\odot 2010$ S. Karger AG, Basel

\section{Introduction}

The anterior and posterior maculae flavae (MFe) are observed clinically at one end of the membranous portion of vocal folds (VF) on endoscopy of the larynx. They form conspicuous mucosal bulges and are visible through the mucosa as whitish yellow masses.

The anterior MFe of the VF have already been described as the nodulus elasticus in the anatomic textbook by Lanz and Wachsmuth [1]. They say that the MFe are elastic nodules located at the anterior end of the VF with numerous elastic fibers [1]. Hirano [2] noted the MFe to be masses of dense elastic fibers at the anterior and posterior ends of the VF, referring to the former as the anterior macula flava (nodulus elasticus) and the latter as the posterior macula flava.

The histological structure of MFe in the human adult vocal fold mucosa (VFM) is unique, and their role in the $\mathrm{VF}$ as a vibrating tissue is interesting. However, their role in the human adult VFM has not been clarified until now

\section{KARGER \\ Fax +4161306 1234 \\ E-Mail karger@karger.ch}

www.karger.com
(C) 2010 S. Karger AG, Basel

$1021-7762 / 10 / 0624-0178 \$ 26.00 / 0$

Accessible online at:

www.karger.com/fpl
Kiminori Sato, $\mathrm{MD}, \mathrm{PhD}$

Department of Otolaryngology-Head and Neck Surgery

Kurume University School of Medicine

67 Asahi-machi, Kurume 830-0011 (Japan)

Tel. +81942 31 7575, Fax +8194237 1200, E-Mail kimisato@ oct-net.ne.jp 


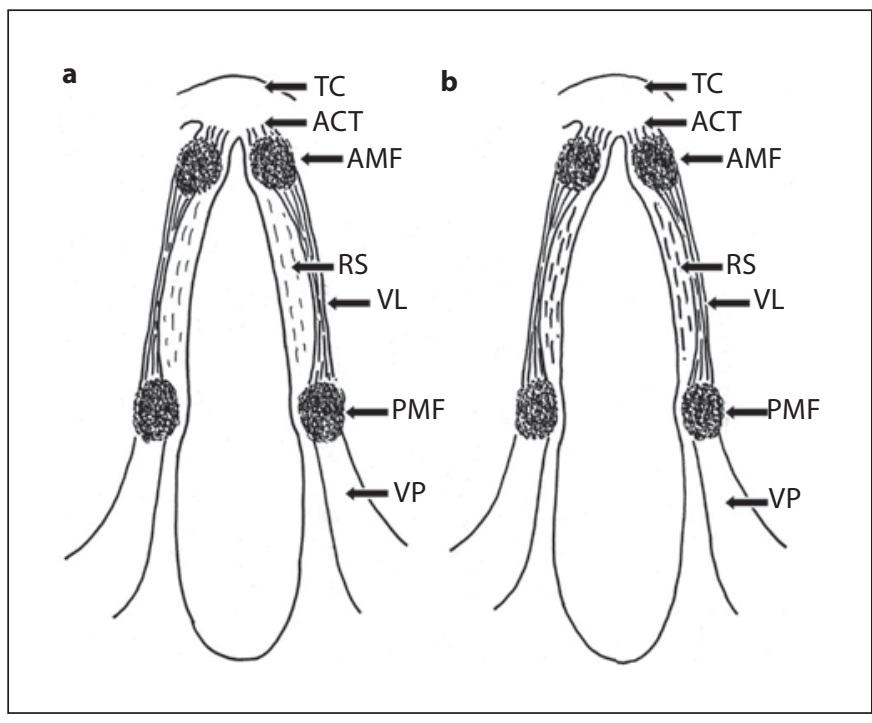

Fig. 1. Human adult MFe and VFM. TC = Thyroid cartilage; $\mathrm{ACT}=$ anterior commissure tendon; $\mathrm{AMF}=$ anterior macula flava; $\mathrm{PMF}=$ posterior macula flava; $\mathrm{VP}=$ vocal process of arytenoid cartilage; $\mathrm{RS}=$ Reinke's space; $\mathrm{VL}=$ vocal ligament. a Young adult. b Aged adult.

[1-7]. During the past decade, we investigated the morphology of the MFe in the human adult VF. The purpose of the present paper is to summarize our current morphological results concerning the MFe in the human adult VFM.

\section{Fine Structure of Adult VFM around MFe (fig. 1a)}

In adults, the VF has a layered structure consisting of the epithelium, the lamina propria (superficial, intermediate and deep layer) and the vocalis muscle $[2,3]$. The superficial layer is referred to as Reinke's space. The structure consisting of the intermediate and deep layers of the lamina propria is called the vocal ligament. The vocal ligament runs between the anterior and posterior MFe. The mucosal portion of the layered structure is based on the differences of extracellular matrix distribution, and it is essential for VF vibration and is required for phonation [2].

The viscoelastic properties of the lamina propria of human VFM, especially its superficial layer (Reinke's space), are essential for the vibrating behavior (phonation) and depend on extracellular matrices (EM) such as collagenous fibers, reticular fibers, elastic fibers, glycoproteins and glycosaminoglycan. Fibrillar collagen serves as a stabilizing scaffold in the EM. Reinke's space, a threedimensional structure of reticular fibers, which consist of fibrillar collagen (type III collagen) and whose slender fibrils form delicate three-dimensional networks, possesses innumerable potential spaces [8]. These extracellular interstitial spaces are made up of minute chambers or compartments occupied by other EM [8]. Glycosaminoglycans and glycoproteins are situated among the spaces of the reticular fibers, and elastic fibers run through the spaces. Hyaluronic acid, one of the glycosaminoglycans, contributes to tissue viscosity and is an important molecule for maintaining optimal tissue properties. Hyaluronic acid also plays an important role in the viscoelasticity of the human VFM [9-11]. The complex of reticular fibers and other EM is important for the viscoelastic properties of the human VFM [8].

The vibratory portion of the VF is connected to the thyroid cartilage anteriorly via the intervening anterior MFe and anterior commissure tendon. Posteriorly, it is joined to the arytenoid cartilage via the intervening posterior MFe [3] (fig. 1).

Fine Structure of Younger Adult MFe (fig. 1a) [12-17]

Human VFM has dense masses of cells and EM, which have been called MFe (fig. 2, 3). The MFe are located at the anterior and posterior ends of the membranous portion of the bilateral VF (fig. 1). They are elliptical in shape and their size is approximately $1.5 \times 1.5 \times 1 \mathrm{~mm}$. The border between the MFe and the surrounding soft tissue is relatively clearly delineated (fig. 2). The EM of MFe are composed of fibrillar protein such as collagenous, reticular, and elastic fibers, glycoprotein, and glycosaminoglycan. These EM in the MFe extend to those in the lamina propria (Reinke's space and the vocal ligament) of the VFM.

EM are usually produced by interstitial cells such as fibroblasts. The distribution of the interstitial cells differs according to site in the human VFM. Their cell density is greater in the MFe and sparse in Reinke's space (lamina propria of the VFM; fig. 4).

Interstitial cells with a starlike appearance in the human MFe were discovered in our previous studies in 2001 [13] (fig. 3, 5). These cells have no nomenclature and are thus designated vocal fold stellate cells (VFSCs) in the series of our study. Many VFSCs are present in the MFe of adult VFM, and their density is high. But none are found in Reinke's space. Fibroblasts can be seen throughout the human adult VFM. However, the fibroblasts are sparse in Reinke's space (lamina propria of the VFM). The density of cells in the adult MFe is about 2.5 times that in Reinke's space (fig. 4).

There are a number of morphological differences between VFSCs and conventional fibroblasts in the human VFM; VFSCs constantly synthesize EM, which is essen- 
Fig. 2. Posterior macula flava (arrows) on coronal section of a human adult VF. a Elastica-van Gieson stain. b Alcian blue stain $(\mathrm{pH} 2.5)$. Original $\times 20$. The posterior macula flava is strongly stained (arrows) with each stain. TAM = Thyroarytenoid muscle; $\mathrm{LG}=$ laryngeal gland.
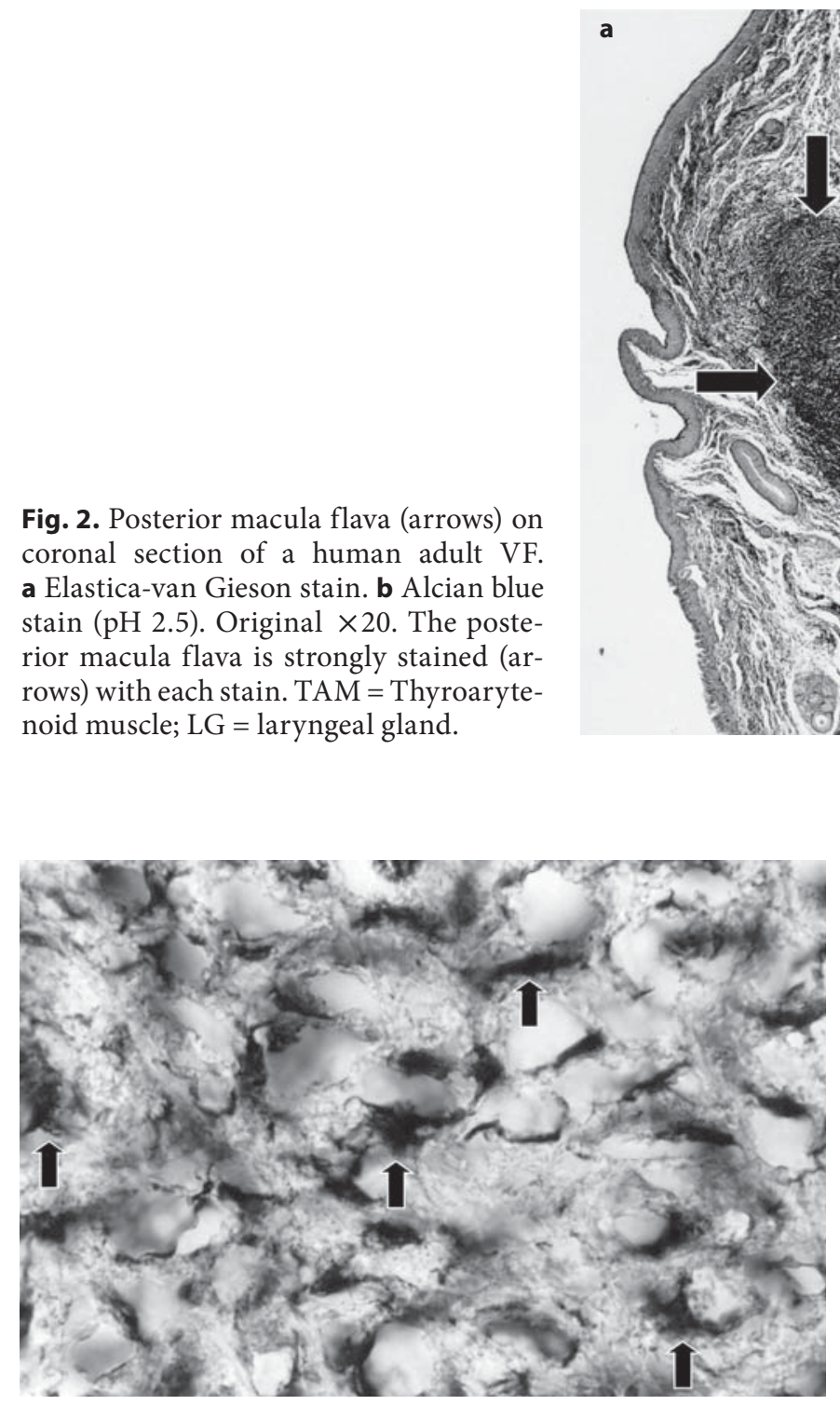

Fig. 3. Light microscopy of the macula flava of a human adult VF. Arrows: VFSCs. Gold chloride method: no counterstaining, original $\times 200$.

tial for the viscoelastic properties of the human VFM. A comparison between VFSCs and conventional fibroblasts is given in table 1 .

The cell density of VFSCs in the human MFe is high (fig. 3-5). VFSCs are irregular and stellate in shape and possess slender cytoplasmic processes. The nucleus-cytoplasm ratio is small and well-developed intracellular organelles such as rough endoplasmic reticulum and Golgi apparatus are present, indicating that these cells are activated and that protein synthesis is occurring within

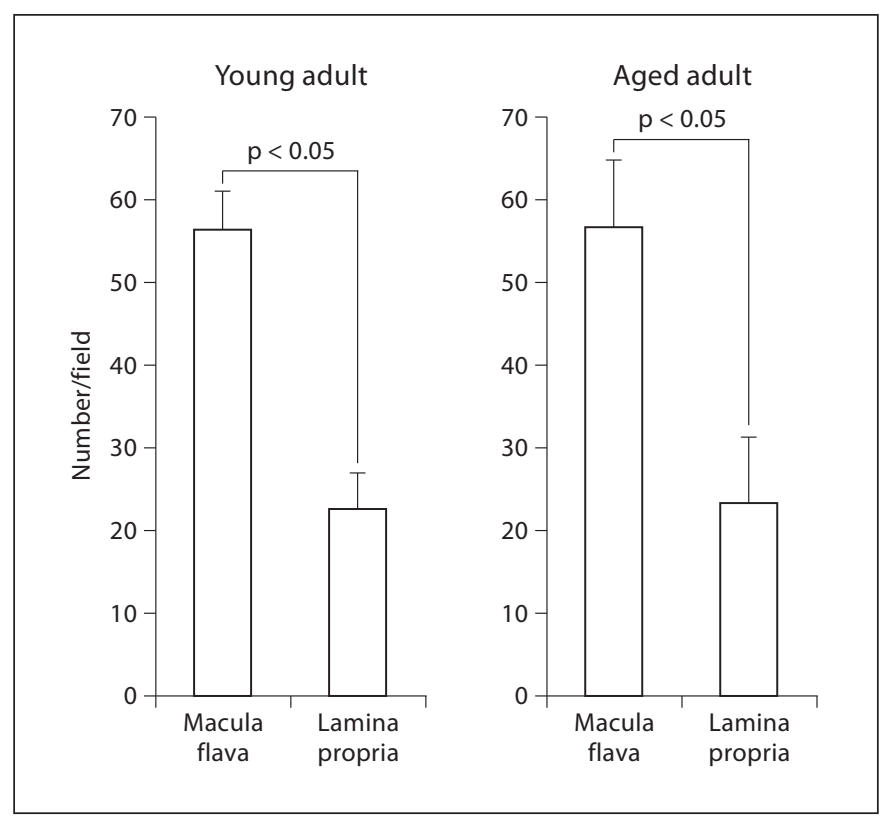

Fig. 4. Cell density in the human adult VFM (data from ref. 16).

them. Along the surface of the VFSCs, a number of vesicles are present. The VFSCs possess lipid droplets and store vitamin A.

It is generally accepted that vitamin A (retinol), an essential liposoluble vitamin, is used as a morphogen that controls the differentiation and morphogenesis of cells. 

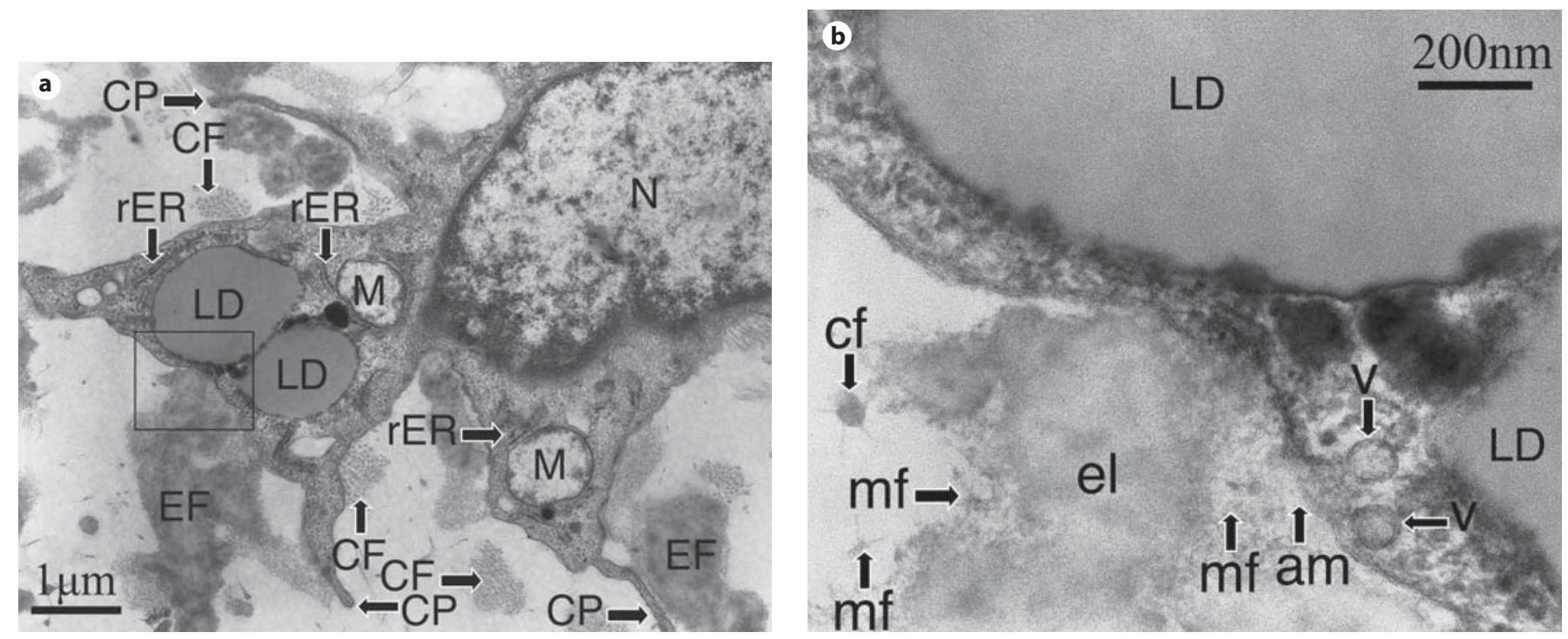

Fig. 5. Transmission electron microscopy of VFSC in the human adult macula flava. Uranyl acetate and lead citrate stain. a Synthesized collagenous and elastic fibers are detected around VFSC. N = Nucleus; LD = lipid droplet; $\mathrm{CP}=$ cytoplasmic process; $\mathrm{rER}=$ rough endoplasmic reticulum; $\mathrm{M}=$ mitochondrion; $\mathrm{CF}=$ collagenous fibers; EF = elastic fibers. $\mathbf{b}$ Higher magnification of frame shown in $\mathbf{a}$. Synthesis of collagenous and elastic fibers by VFSC. $\mathrm{v}=$ Vesicle; $\mathrm{am}=$ amorphous material; $\mathrm{mf}=$ microfibril; $\mathrm{cf}=$ collagen fibril; el = elastin.

Table 1. Comparison between VFSCs in the macula flava and fibroblasts in human VFM

\begin{tabular}{lll}
\hline & VFSCs & Fibroblasts \\
\hline Location & macula flava & membranous portion of VFM \\
Cell density & high & low \\
Shape & stellate & spindle, oval \\
Cytoplasmic process & present & not present \\
Nucleus-cytoplasm ratio & small & large \\
Intracellular organelle & well developed & poor \\
Rough endoplasmic reticulum and Golgi apparatus & well developed & poor \\
Vesicles & many & few \\
Lipid droplets & many & few \\
Vitamin A storing & stored & not stored \\
Synthesis of extracellular matrices & much & little \\
Collagenous fibers around cells & many & not very many \\
Reticular fibers around cells & many & not very many \\
Elastic fibers around cells & many & not very many \\
Hyaluronic acid around cells & much & not much \\
Periodic acid-Schiff stain & strong & weak \\
Type III collagen stain & strong & weak \\
CD44 stain & strong & weak \\
Radiation sensitivity & higher than fibroblasts & \\
\hline
\end{tabular}

Vitamin A strongly influences the activity of adenosine triphosphate sulfurylase, and is related to the synthesis of glycosaminoglycan [18-20]. In porcine VF, increased exposure to retinol induces sequential reorganization of the actin cytoskeleton in activated VFSCs [21]. On the other hand, vitamin A is not essential to maintaining the EM of the rat VF [22]. The role of vitamin A in VFSCs is ambiguous. 
There are many collagenous fibers that stain red with elastica-van Gieson staining (fig. 2a) and reticular fibers around VFSCs in the human adult MFe. There are many vesicles at the periphery of cytoplasm, and newly released amorphous materials are present on the cell surface of VFSCs (fig. 5b). Microfibrils 10-15 nm wide are observed around the amorphous material. Collagen fibrils are detected near the microfibrils (fig. 5b). Collagenous fibers are made up of several collagen fibrils. Thus, the VFSCs constantly synthesize not only collagenous fibers, but also reticular fibers in the human adult MFe.

Many elastic fibers that stain black with elastica-van Gieson staining (fig. 2a) are present around VFSCs in the human adult MFe. There are many vesicles at the periphery of the cytoplasm, and newly released amorphous materials are seen on the cell surface of VFSCs (fig. 5b). Microfibrils 10-15 $\mathrm{nm}$ wide are situated around the amorphous material. There are microfibril assemblies on which elastin is deposited (fig. 5b). The amorphous substance of elastic fibers is produced by fusion of microfibrils. The elastic fibers consist of amorphous substance and microfibrils. The VFSCs constantly synthesize elastic fibers in the human adult MFe.

Hyaluronic acid, one of the glycosaminoglycans, plays an important role in the viscoelasticity of the human VFM [9-11]. MFe are strongly stained light blue with Alcian blue at $\mathrm{pH} 2.5$ (fig. 2b), while the ground substance in the MFe that is strongly stained with Alcian blue is digested by hyaluronidase. Much hyaluronic acid is located around the VFSCs in the human adult MFe (fig. 2b). The VFSCs constantly synthesize hyaluronic acid in the human adult MFe.

CD44 is a cell membrane-localized receptor for hyaluronic acid. It is an interesting question how cells organize their EM and how these matrices feed back to cell metabolism involving specific cell-matrix interactions, which are mediated by cell surface matrix receptors. The percentage of CD44-positive cells in the MFe of younger adults is high, and most of the VFSCs $(96.3 \pm 1.8 \%$, mean $\pm \mathrm{SD}$ ) are stained with CD44 [16]. On the other hand, CD44-positive fibroblasts in Reinke's space are sparse $(6.8 \pm 4.6 \%)$ [16]. VFSCs in the MFe and CD44 both play important roles in the metabolism of hyaluronic acid in the human VFM.

Regarding radiation-induced damage to human $\mathrm{MFe}$ and VFSCs [17], the MFe components of the irradiated VFM are the same as those in a normal VF. However, irradiated MFe have less fibers and ground substance components than normal human adult MFe. Fibroblasts in Reinke's space show no morphologic changes after irra- diation. On the other hand, most of the VFSCs in the irradiated MFe have dark cytoplasm. The nucleus-cytoplasm ratio is relatively small, but there are few intracellular organelles in the cytoplasm. Some VFSCs show degeneration. Fewer vesicles are present at the periphery of the cytoplasm, and newly released amorphous materials from the vesicles are less than normal. Elastic and collagenous fibers immediately surrounding VFSCs in the irradiated $\mathrm{MFe}$ are lower in number than usual, but elastic fibers and collagenous fibers composed of irregular collagen fibrils can be detected at a distance from VFSCs. This suggests that radiation inhibits the synthesis of precursors of collagenous and elastic fibers by VFSCs. Radiation sensitivity of VFSCs is higher than that of conventional fibroblasts in Reinke's space, and irradiated VFSCs decrease their level of activity.

Human VFSCs in the MFe were isolated and subcultured, and the morphological differences between human VFSCs and conventional fibroblasts were compared [23]. Results showed that subcultured cells of the MFe are stellate in shape and have cytoplasmic processes. They are larger than conventional fibroblasts. Human VFSCs proliferate by attaching their cytoplasmic processes to each other. In isolation and during a 7- to 10-month subculture period, human VFSCs continue to exhibit the same morphological characteristics, which differ from those of conventional fibroblasts.

The histological structure of the lamina propria of VFM significantly varies between animals [24]. Furthermore, the structure of other mammalian VFM is different from that of humans [24]. In animals the MFe are also present at the anterior and posterior ends of the membranous portion of the VF. But there is no structure equivalent to the human vocal ligament or the layered structure of the human VF [24]. For example, the structure and morphological functions of the canine MFe are found to differ from those of human adult MFe [25]. There have been some investigations of MFe using animal samples [21, 22, 26]. Since the human VF differs in histology, physiology and pathology from that of other mammals, MFe should ideally be collected and investigated from human samples.

To summarize, our current studies indicate that the VFSCs in young adult human MFe are not quiescent but active and constantly synthesize EM essential for the viscoelasticity of the human VFM. It is also indicated that the VFSCs in the adult MFe are connected with fibrogenesis and fibrolysis, and produce necessary EM that maintain the viscoelastic properties of the human VFM. From our current studies it can be inferred that the VFSCs in the MFe form an independent cell category that should 


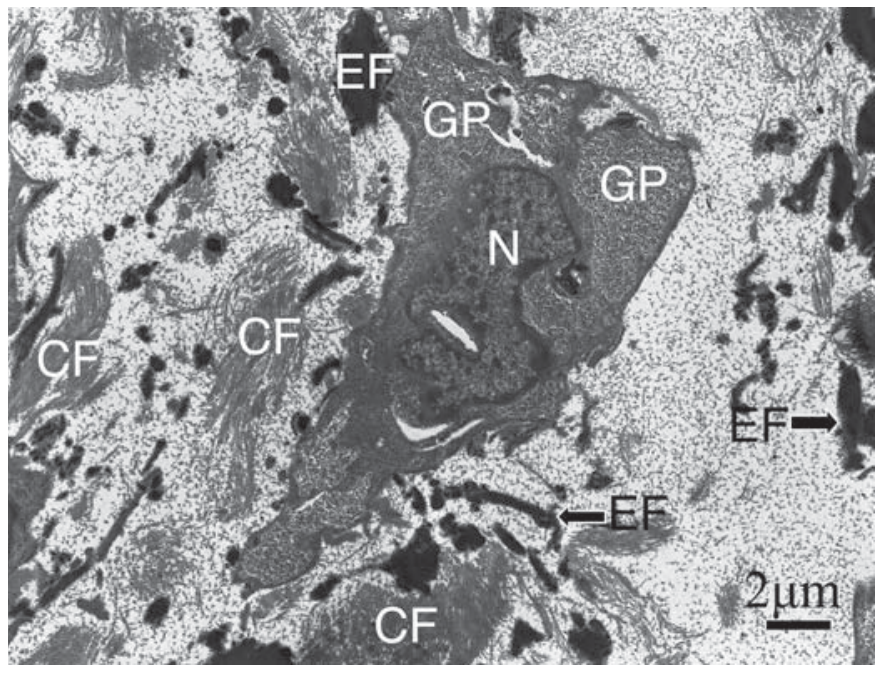

Fig. 6. Transmission electron microscopy of VFSC in the human aged adult macula flava (79-year-old male). Tannic acid stain. $\mathrm{N}$ = Nucleus; $\mathrm{GP}=$ glycogen particles; $\mathrm{CF}$ = collagenous fibers; $\mathrm{EF}=$ elastic fibers.

be considered a new category of cells and are involved in the metabolism of EM in the human VFM.

Fine Structure of Aged MFe and VFM (fig. 1b) [27, 28]

The voice changes with aging. Actually, EM have changed in the aged VFM [29-31]. Changes in the threedimensional structure of collagenous and reticular fibers, as well as their qualitative and quantitative changes, have an effect on the three-dimensional structure of the EM [31]. In addition, age-related changes in other EM constituents, such as elastic fibers and glycosaminoglycan, among the collagenous and reticular fibers, influence the three-dimensional structure of the EM [30,31]. Thus, viscoelasticity must change to ensure normal phonation, and this change in viscoelasticity explains one component of aging of the voice.

The MFe of aged adult VFM are dense masses of VFSCs and EM, and their components are the same as in younger adult MFe. Many VFSCs are present in the MFe of aged adult VFM, and their density is high. The density of aged adult VFSCs is the same as that of younger adult VFSCs (fig. 4). The density of VFSCs in the MFe is about 2.5 times that of fibroblasts in Reinke's space (fig. 4).

Basically, the morphological characteristics of aged VFSCs are the same as those of younger adults. The most typical findings in the MFe of aged VF are the morphologic changes in the VFSCs. The nucleus-cytoplasm ratio is relatively small, but there are fewer intracellular organ- elles, such as rough endoplasmic reticulum and Golgi apparatus, than in younger adults, indicating that aged VFSCs are not activated and have no constant protein synthesis. Consequently, there are few vesicles at the periphery of the cytoplasm of aged VFSCs, and there are few newly released amorphous materials, indicating that the number of fibers synthesized by these inactivated cells have decreased. Some aged adult VFSCs have degenerated, and an accumulation of glycogen particles is seen in the cytoplasm (fig. 6). In tannic acid-stained material, the glycogen particles are electron-dense and approximately $15-30 \mathrm{~nm}$ in diameter. In material stained with uranyl acetate and lead citrate, a collection of glycogen particles is present as a lucent stained area (glycogen lake) within the cytoplasm. There is an inverse relationship between the metabolic activities of cells and glycogen deposits, and glycogen tends to accumulate in atrophic cells and in cells that are presumably less active [32]. Many lipofuscin granules are noted around the lipid droplets in the aged adult VFSCs. Lipofuscin granules are regarded as residual bodies left behind in the cell after lysosomal activity, and they are derived from the degradation of endogenous, and not exogenous material in the lysosome [32]. The findings mentioned above are recognized to various degrees.

There are not as many vesicles at the periphery of the cytoplasm of aged adult VFSCs, and especially few vesicles can be detected at the periphery of the cytoplasm of degenerated VFSCs. Newly released amorphous materials from the vesicles are present on the cell surface of VFSCs, but not as much as in younger adults. There are collagenous, reticular and elastic fibers around VFSCs in aged adult MFe. The synthesis of these fibers takes place in the same way as in younger adult MFe. However, the number of fibers synthesized by inactivated VFSCs has decreased.

A large amount of hyaluronic acid is situated around VFSCs in aged adult human MFe, as in younger adults. The percentage of CD44-positive cells in the MFe of aged adults is high, and most of the VFSCs $(96.9 \pm 1.4 \%)$ are stained with CD44 [16]. On the other hand, CD44-positive cells are sparse $(7.3 \pm 3.1 \%)$ among the fibroblasts in Reinke's space of aged adults [16]. VFSCs in the MFe and CD44 both play important roles in the metabolism of hyaluronic acid in aged human VFM.

To summarize, adult aged MFe are also composed of dense masses of VFSCs and EM such as collagenous, reticular, and elastic fibers, glycoprotein, and glycosaminoglycan (hyaluronic acid). However, VFSCs in the aged MFe decrease their activity, have abnormal metabolism, and undergo degeneration. Age-related changes in the 
VFSCs of MFe are believed to influence the metabolism of EM in the VFM, as well as the viscoelasticity of aged VFM, and are one of the causes of aging of the voice.

\section{Conclusion}

Human adult MFe are composed of dense masses of VFSCs and EM such as collagenous, reticular, and elastic fibers, glycoprotein, and glycosaminoglycan (hyaluronic acid). These EM are essential for the human VFM as a vibrating tissue. The VFSCs in the human adult MFe form an independent cell category that should be considered a new category of cells in the human VF.
On the basis of the results of our current study, human MFe located at both ends of the VFM are believed to be involved in the metabolism of EM essential for the viscoelastic properties of the lamina propria of the adult human VFM. Human adult MFe are considered to be responsible for maintaining the characteristic layered structure of the human VFM. Adult human MFe are also considered to be an important structure in the aging of human VFM.

Our current morphological results concerning the MFe in the adult human VFM are summarized. More research, especially using various methods, is needed to validate the significance and roles of the human MFe.

\section{References}

1 Lanz TV, Wachsmuth W: Praktische Anatomie Hals. Berlin, Springer, 1955.

2 Hirano M: Phonosurgery: basic and clinical investigations. Otologia (Fukuoka) 1975; 21(suppl 1):239-260.

3 Hirano M, Sato K: Histological Color Atlas of the Human Larynx. San Diego, Singular Publishing Group, 1993.

4 Subotic R, Vecerina S, Krajina Z, Hirano M, Kurita S: Histological structure of vocal fold lamina propria in foetal larynx. Acta Otolaryngol (Stockh) 1984;97:403-406.

$\checkmark 5$ Vecerina-Volic S, Hirano M, Karovic-Krzelj V: Macula flava in the vocal fold of human fetus. Acta Otolaryngol (Stockh) 1988;105: 144-148.

-6 Campos Banales ME, Perez Pinero B, Rivero J, Ruiz Casal E, Lopez Aguado D: Histological structure of the vocal fold in the human larynx. Acta Otolaryngol (Stockh) 1995;115: 701-704.

$\checkmark 7$ Fayoux P, Devisme L, Merrot O, Chevalier D, Gosselin B: Histologic structure and development of the laryngeal macula flava. Ann Otol Rhinol Laryngol 2004;113:498-504.

$>8$ Sato K: Reticular fibers in the vocal fold mucosa. Ann Otol Rhinol Laryngol 1998;107: 1023-1028.

9 Matsuo K, Watanabe S, Hirano M, Kamimura M, Tanaka Y, Takazono I: Acid mucopolysaccharide and glycoprotein in the vocal fold: alterations with aging. Pract Otol (Kyoto) $1984 ; 77: 817-822$.

10 Gray SD, Titze IR, Chan R, Hammond TH: Vocal fold proteoglycans and their influence on biomechanics. Laryngoscope 1999;109: 845-854.

$\checkmark 11$ Chan RW, Gray SD, Titze IR: The importance of hyaluronic acid in vocal fold biomechanics. Otolaryngol Head Neck Surg 2001; 124:607-614.
12 Sato K, Hirano M: Histologic investigation of the macula flava of the human vocal fold. Ann Otol Rhinol Laryngol 1995;104:138143.

13 Sato K, Hirano M, Nakashima T: Stellate cells in the human vocal fold. Ann Otol Rhinol Laryngol 2001;110:319-325.

14 Sato K, Hirano M, Nakashima T: Vitamin Astoring stellate cells in the human vocal fold. Acta Otolaryngol 2003;123:106-110.

15 Sato K, Hirano M, Nakashima T: 3D struc ture of the macula flava in the human vocal fold. Acta Otolaryngol 2003;123:269-273.

16 Sato K, Sakamoto K, Nakashima T: Expres sion and distribution of CD44 and hyaluronic acid in human vocal fold mucosa. Ann Otol Rhinol Laryngol 2006;115:741-748.

17 Sato K, Shirouzu H, Nakashima T: Irradiated macula flava in the human vocal fold mucosa. Am J Otolaryngol 2008;29:312-318.

18 Sundaresan PR: Vitamin A and the sulfate activating enzymes. Biochim Biophys Acta 1966;113:95-109.

19 DeLuca L, Wolf G: Effect of vitamin A on the mucopolysaccharides of lung tissue. Arch Biochem Biophys 1968;123:1-8.

20 Levi AS, Geller S, Root DM, Wolf G: The effect of vitamin A and other dietary constituents on the activity of adenosine triphosphate sulphurylase. Biochem J 1968;109: 69-74.

21 Fuja TJ, Probst-Fuja MN, Titze IR: Changes in expression of extracellular matrix genes, fibrogenic factors, and actin cytoskeletal organization in retinol treated and untreated vocal fold stellate cells. Matrix Biol 2006;25: 59-67.

-22 Tateya T, Tateya I, Surles RL, Tanumihardjo S, Bless DM: Roles of vitamin A and macula flava in maintaining vocal folds. Ann Otol Rhinol Laryngol 2008;117:65-73.
23 Sato K, Miyajima Y, Izumaru S, Nakashima $\mathrm{T}$ : Cultured stellate cells in human vocal fold mucosa. J Laryngol Otol 2008;122:13391342 .

24 Kurita S, Nagata K, Hirano M: Comparative histology of mammalian vocal folds; in Kirchner JA (eds): Vocal Fold Histopathology. San Diego, College Hill Press, 1986, pp $1-10$.

-25 Sato K, Hirano M, Nakashima T: Comparative histology of the maculae flavae of the vocal folds. Ann Otol Rhinol Laryngol 2000; 109:136-140.

26 Fuja TJ, Probst-Fuja MN, Titze IR: Transdifferentiation of vocal-fold stellate cells and all-trans retinol-induced deactivation. Cell Tissue Res 2005;322:417-424

27 Sato K, Hirano M: Age-related changes of the macula flava of the human vocal fold. Ann Otol Rhinol Laryngol 1995;104:839-844.

28 Sato K, Hirano M, Nakashima T: Age-related changes in vitamin A-storing stellate cells of human vocal folds. Ann Otol Rhinol Laryngol 2004;113:108-112.

29 Hirano M, Kurita S, Sakaguchi S: Ageing of the vibratory tissue of human vocal folds. Acta Otolaryngol (Stockh) 1989;107:428433.

30 Sato K, Hirano M: Age-related changes of elastic fibers in the superficial layer of the lamina propria of vocal folds. Ann Otol Rhinol Laryngol 1997;106:44-48.

-31 Sato K, Hirano M, Nakashima T: Age-related changes of collagenous fibers in the human vocal fold mucosa. Ann Otol Rhinol Laryngol 2002;111:15-20.

32 Ghadially FN: Ultrastructural Pathology of the Cell and Matrix. London, Butterworths, 1988, pp 608-613, pp 962-969. 